\title{
PERCEPÇÃO DO IMPACTO NO TRABALHO DE UM TREINAMENTO ON- LINE SOBRE PREVENÇÃO DE LESÃO POR PRESSÃO ${ }^{1}$
}

\author{
Juscilynne Barros da Costa Aroldi², Heloisa Helena Ciqueto Peres ${ }^{3}$, Vera Lucia Mira ${ }^{4}$
}

\begin{abstract}
${ }^{1}$ Artigo extraído da tese - Treinamento on-line sobre úlcera por pressão: aprendizagem, reação e o impacto no trabalho, apresentada ao Programa de Pós-Graduação em Gerenciamento em Enfermagem da Escola de Enfermagem da Universidade de São Paulo (USP), em 2016.

2 Doutora em Ciências. Enfermeira Auditora do Exército Brasileiro. São Paulo, São Paulo, Brasil. E-mail: juscilynne@gmail.com

${ }^{3}$ Doutora em Enfermagem. Professora do Departamento de Orientação Profissional da Escola de Enfermagem da USP. São Paulo, São Paulo, Brasil. E-mail: hhcperes@gmail.com

${ }^{4}$ Doutora em Enfermagem. Professora do Departamento de Orientação Profissional da Escola de Enfermagem da USP. São Paulo, São Paulo, Brasil. E-mail: vlmirag@usp.br
\end{abstract}

\section{RESUMO}

Objetivo: avaliar a percepção do impacto em amplitude e o suporte à transferência do treinamento on-line sobre prevenção de lesão por pressão.

Método: estudo exploratório-descritivo, desenvolvido no município de São Paulo, no Hospital Universitário da Universidade de São Paulo. A população da pesquisa abrangeu 97 enfermeiros que atuavam em unidades com pacientes de maior risco de desenvolvimento de lesão por pressão. Além disso, foram aplicados dois instrumentos para avaliação de suporte à transferência e de percepção do impacto. Os dados foram submetidos às análises estatísticas descritivas e coeficientes de correlação de Pearson, com auxílio do software $\mathrm{R}^{\circledR} 3.2 .2$. $\mathrm{O}$ coeficiente Alpha de Cronbach foi utilizado para verificar a consistência interna das escalas.

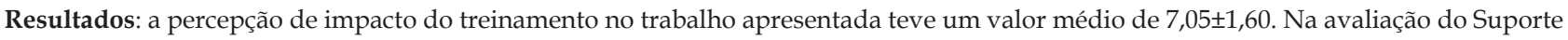
à Transferência, a média foi de 5,82 $\pm 1,45$. A análise de impacto no trabalho evidenciou valores significativos, indicando que houve transferência e construção de conhecimentos, bem como habilidades desenvolvidas pelos enfermeiros no treinamento on-line para a prática assistencial na prevenção de lesão por pressão. A avaliação do suporte à transferência permitiu examinar o tipo de ambiente encontrado pelo enfermeiro para fazer com que os conteúdos assimilados fossem transferidos para sua prática profissional.

Conclusão: os resultados das avaliações evidenciam que o treinamento on-line agrega conhecimento aos enfermeiros e contribuem para a implementação de novas tecnologias educacionais nas ações permanentes de treinamento e desenvolvimento dos profissionais da enfermagem.

DESCRITORES: Úlcera por pressão. Educação à distância. Tecnologia educacional. Educação continuada em enfermagem. Capacitação. Informática em enfermagem.

\section{IMPACT PERCEPTION AT WORK FROM AN ONLINE TRAINING ON THE PREVENTION OF PRESSURE INJURY}

\begin{abstract}
Objective: to assess the impact perception in amplitude, and the support for the transferring of the online training on pressure injury prevention.

Method: this is an exploratory-descriptive study, developed in the city of São Paulo, in the University Hospital of the University of São Paulo. The research population was composed of 97 nurses who worked in units with patients at higher risk of developing pressure injury. In addition, two instruments were applied for assessing the support on the transfer and the impact perception. The data were submitted to descriptive statistical analyzes and Pearson's correlation coefficients, with the aid of the software ${ }^{\circledR}$ 3.2.2. Cronbach's Alpha coefficient was used to verify the internal consistency of the scales.

Results: the impact perception of the training in the current study had an average value of $7.05 \pm 1.60$. In the assessment of the Transfer Support, the average was $5.82 \pm 1.45$. The impact analysis on the study showed significant values, indicating that there was transference and construction of knowledge, as well as skills developed by nurses in the online training for the care practice in the prevention of pressure injury. The assessment of the transfer support allowed us to examine the type of environment found by nurses to make the assimilated contents transfer to their professional practice.

Conclusion: the results of the assessments show that the online training provides knowledge to the nurses and contribute to the implementation of new educational technologies in the permanent actions of training and development of the nursing professionals.

DESCRIPTORS: Pressure ulcer. Distance education. Educational technology. Continuing Education in nursing. Training. Informatics in nursing.
\end{abstract}




\section{PERCEPCIÓN DEL IMPACTO EN EL TRABAJO DE UN ENTRENAMIENTO ON-LINE SOBRE PREVENCIÓN DE LESIÓN POR PRESIÓN}

\section{RESUMEN}

Objetivo: evaluar la percepción del impacto en amplitud y el soporte a la transferencia del entrenamiento en línea sobre prevención de lesión por presión.

Método: estudio exploratorio-descriptivo, desarrollado en el municipio de São Paulo, en el Hospital Universitario de la Universidad de São Paulo. La población de la encuesta abarcó a 97 enfermeros que actuaban en unidades con pacientes de mayor riesgo de desarrollo de lesión por presión. Además, se aplicaron dos instrumentos para evaluar el apoyo a la transferencia y la percepción del impacto. Los datos fueron sometidos a los análisis estadísticos descriptivos y coeficientes de correlación de Pearson, con ayuda del software R® 3.2.2. El coeficiente Alpha de Cronbach fue utilizado para verificar la consistencia interna de las escalas.

Resultados: la percepción de impacto del entrenamiento en el trabajo presentada tuvo un valor promedio de 7,05 $\pm 1,60$. En la evaluación del soporte a la transferencia, la media fue de 5,82 $\pm 1,45$. El análisis de impacto en el trabajo evidenció valores significativos, indicando que hubo transferencia y construcción de conocimientos, así como habilidades desarrolladas por los enfermeros en el entrenamiento on-line para la práctica asistencial en la prevención de lesión por presión. La evaluación del soporte a la transferencia permitió examinar el tipo de ambiente encontrado por el enfermero para hacer que los contenidos asimilados fueran transferidos a su práctica profesional.

Conclusión: los resultados de las evaluaciones evidencian que el entrenamiento on-line agrega conocimiento a los enfermeros y contribuyen a la implementación de nuevas tecnologías educativas en las acciones permanentes de entrenamiento y desarrollo de los profesionales de la enfermería.

DESCRIPTORES: Úlcera por presión. Educación a distancia. Tecnología educacional. Educación continuada en enfermería. Capacitación. Informática aplicada a la enfermería.

\section{INTRODUÇÃO}

O desenvolvimento das profissões tem avançado consideravelmente, sendo necessários a atualização contínua do conhecimento e o aprimoramento de competências para que o profissional possa conviver com a diversidade do conhecimento. ${ }^{1}$

A formação do profissional tem sido uma importante aliada para instituições de diversas áreas, entre as quais aquelas que prestam serviço à saúde, uma vez que estas, cada vez mais, almejam trabalhadores com amplo conhecimento e aptos a resolverem problemas. ${ }^{2}$ Assim, as organizações, cada vez mais integradas ao uso das novas tecnologias, têm buscado capacitar seus profissionais por meio de uma educação reflexiva e colaborativa. ${ }^{3}$

As Tecnologias da Informação e Comunicação (TICs) são definidas como ferramentas para facilitar a comunicação, o processamento e a transmissão da informação por meios eletrônicos. Esta definição engloba, por exemplo, o uso do rádio, do telefone (fixo e móvel), de computadores e da internet. ${ }^{4}$

O desenvolvimento e utilização das novas TICs, em treinamentos nas organizações, são, desse modo, essenciais para facilitar a aquisição e atualização de conhecimentos dos indivíduos. ${ }^{5}$

As organizações, por sua vez, têm utilizado as TICs para o desenvolvimento de estratégias de atualização contínua de competências organizacionais e individuais, sendo necessária uma mudança de comportamento, uma vez que a aprendizagem deve estar orientada para a colaboração. O ensino e a aprendizagem devem, nesse sentido, se realizar de forma colaborativa, destacando-se, para tanto, a importância de que cada profissional conheça seu papel e possa contribuir de forma adequada e eficaz nesse processo. ${ }^{6-9}$

Assim, compreende-se a educação para profissionais como um processo dinâmico e contínuo da construção do conhecimento, através do pensamento livre e da consciência crítico-reflexiva, para a criação do compromisso pessoal e profissional na transformação da (e na ação sobre a) realidade. ${ }^{10}$

Dito isto, é importante salientar que, na área da Psicologia Social e do Trabalho define-se Treinamento, Desenvolvimento e Educação (TD\&E) da seguinte forma: ${ }^{11}$

Treinamento - utilizado para apoiar os indivíduos na aquisição de uma nova habilidade e um novo conhecimento. São eventos educacionais de curta e média duração, que visam à melhoria do desempenho funcional, por meio da criação de situações que facilitem a aquisição, a retenção e a transferência da aprendizagem para as atividades do trabalho. ${ }^{11}$

Desenvolvimento - processo de aprendizagem destinado ao crescimento do indivíduo. É o conjunto de experiências e oportunidades de aprendizagem, proporcionadas pela organização e que apoiam o crescimento pessoal do empregado por meio de programas de autodesenvolvimento. ${ }^{11-12}$

Educação - forma mais ampla de aprendizagem por meio de programas ou conjuntos de eventos educacionais de média e longa duração que visam à formação e à qualificação profissional contínuas dos empregados. ${ }^{11}$

Apesar de conceitos distintos, Treinamento e 
Desenvolvimento estão intimamente relacionados, não havendo limites claros entre eles, pois o mesmo evento educativo pode ser um treinamento para uns e desenvolvimento para outros. ${ }^{13}$ Assim, é comum o emprego genérico do termo Treinamento para designar ações estruturadas para aquisição e retenção de conhecimentos, habilidades e atitudes. ${ }^{14}$

Dos quatro elementos constitutivos de TD\&E, a avaliação de necessidades de aprendizagem, o planejamento, a execução do programa, e a avaliação de resultados- destacamos a fragilidade deste último, demonstrada pela lacuna nas publicações científicas na área da saúde no Brasil, em que as técnicas aplicadas estão relacionadas mais aos aspectos da avaliação de reação e de aprendizagem. Poucos estudos avaliam o impacto do processo educativo. A adoção de um referencial teórico e metodológico para nortear o processo de avaliação é, desse modo, imprescindível. ${ }^{15}$

Para tanto, a avaliação de treinamento envolve um processo sistemático de levantamento de informações para aperfeiçoar as atividades educativas formais para profissionais. Os principais objetivos da avaliação de treinamento são: controlar o processo, retroalimentar o sistema, tomar decisões sobre o treinamento e torná-lo capaz de promover modificações no ambiente. ${ }^{16}$

De acordo com o referencial adotado nesta pesquisa, a avaliação de resultados de treinamento abordam três níveis de avaliação: ${ }^{17}$ reação que corresponde ao grau de satisfação dos participantes em relação ao treinamento, no que se refere, entre outros, à programação, à aplicabilidade e utilidade do conteúdo e ao ministrante; aprendizagem que demonstra aquisição ou aumento e retenção do conteúdo do curso, medida em testes ou provas de conhecimentos aplicados pelo instrutor ao final do treinamento; ${ }^{18}$ e impacto que verifica a aplicação na prática profissional daquilo que foi assimilado.

Pesquisas recentes sobre avaliação de treinamentos na saúde, entretanto, apontam que o modelo de avaliação deve ser mais amplo, a fim de identificar as variáveis intervenientes ao processo. ${ }^{19-23}$ Além disso, para analisar o impacto das TIC na aprendizagem, importantes autores consideram que existe a necessidade de desenvolver pesquisas com abordagens mais complexas, rigorosas e multifacetadas. ${ }^{24-25}$

A utilização de recursos digitais pode contribuir significativamente para a aprendizagem. No entanto, as publicações são escassas e novos estudos são necessários para identificar o impacto da aplicação desses recursos no processo de aprendizagem dos profissionais de saúde e de enfermagem. ${ }^{26}$
Considerando, assim, a importância das TICs na aprendizagem e avaliação de resultados de treinamentos, é essencial o desenvolvimento de pesquisas com ênfase na avaliação de treinamento on-line de forma mais ampla e que possam identificar o impacto dos treinamentos na assistência.

Nesse contexto, o Modelo Integrado de Avaliação do Treinamento no Trabalho (IMPACT) foi desenvolvido na área da Psicologia Organizacional, para avaliar treinamentos em organizações. ${ }^{18}$

O Impacto em Amplitude ou Impacto do Treinamento no Trabalho compreende o conceito de transferência de aprendizagem e da influência que a ação instrucional é capaz de exercer sobre o desempenho global do treinando, no que se refere à aplicação correta, no ambiente de trabalho, de conhecimentos, habilidades ou atitudes adquiridas em situações de treinamento. ${ }^{18}$

A aplicação daquilo que é aprendido no cotidiano, no entanto, não depende, exclusivamente, do treinando, pois é preciso verificar as condições de trabalho e os recursos disponíveis. Tal processo é denominado como Suporte à Transferência. ${ }^{27}$

O Suporte à Transferência permite avaliar o apoio que o participante do treinamento recebeu para que este aplique no trabalho as novas habilidades adquiridas, referentes aos fatores: Suporte Psicossocial (ou Suporte Gerencial e Social) à Transferência e Suporte Material à Transferência. ${ }^{27}$

O Suporte Psicossocial avalia o apoio gerencial, social e organizacional à aplicação das novas aprendizagens no trabalho, sendo composta por dois conjuntos de itens: fatores situacionais de apoio e consequências associadas à aplicação de novas habilidades no trabalho. ${ }^{27}$

O Suporte Material tem como objetivo avaliar a qualidade, quantidade e disponibilidade de recursos materiais e financeiros, assim como a qualidade e adequação do ambiente físico do local de trabalho. ${ }^{27}$

O conhecimento das variáveis relacionadas ao processo de avaliação dos resultados de treinamento e desenvolvimento contribui para o planejamento e implementação de ferramentas e programas instrucionais que fortaleçam a formação e a prática profissional, iniciando pela avaliação de necessidades de aprendizagem.

Espera-se, desse modo, que os resultados dessa pesquisa contribuam para o aprofundamento do saber sobre a complexidade que envolve os treinamentos na área da Enfermagem, substanciando a construção de metodologia de avaliações de treinamento na área da saúde e possibilitando mudanças 
positivas nas práticas da saúde, na perspectiva da assistência de enfermagem. Destaca-se, dessa maneira, a relevância da temática Lesões por Pressão (LP) para avaliação do treinamento on-line.

No que se refere, especificamente à temática de $\mathrm{LP}$, a literatura aborda a necessidade das instituições de saúde implementarem ações permanentes de treinamento e desenvolvimento aos profissionais, visando a diminuição das taxas de incidência e prevalência das LP. Os estudos, em geral, apontam a importância da capacitação e da utilização de protocolos para avaliação de risco, prevenção e tratamento das LP para melhorar a qualidade da assistência aos pacientes, minimizar o sofrimento, a dor, o tempo de internação e os riscos de infecção, bem como diminuir, por conseguinte, os gastos com o tratamento. ${ }^{28-29}$

Relevando, desta forma, a importância das TICs e avaliação das ações educativas e a necessidade de aprendizagem relativa à LP, essa pesquisa teve como objetivos avaliar a percepção do impacto em amplitude do treinamento on-line sobre Prevenção de Lesão por Pressão e avaliar o suporte à transferência.

\section{MÉTODO}

A pesquisa quantitativa com desenho exploratório-descritivo ocorreu no município de São Paulo, no Hospital Universitário da Universidade de São Paulo (HU/USP), por envolver, sobretudo, uma instituição pública que promove ensino e pesquisa, visando à capacitação dos enfermeiros na temática para melhorar a prática assistencial.

O HU/USP compõe o Sistema Integrado de Saúde da Universidade de São Paulo e tem por finalidade promover o ensino, a pesquisa e a extensão de serviços à comunidade. Localizado no campus da Universidade de São Paulo (USP), na Zona Oeste da cidade deSão Paulo, o HU/USP dispõe de 258 leitos, atua em áreas gerais como Clínica Médica, Cirurgia, Obstetrícia e Ginecologia, Ortopedia e Pediatria. Constitui o campo de ensino e pesquisa das Faculdades de Medicina, Saúde Pública, Enfermagem, Farmácia, Odontologia e Psicologia. A população atendida pelo hospital é constituída pela comunidade USP, que compreende docentes, discentes e servidores da Universidade, incluindo seus dependentes e também pela comunidade residente na região Oeste da cidade de São Paulo, que tem como referência as Unidades Básicas de Saúde dessa região.

A população da pesquisa abrangeu 97 enfermeiros que atuam em unidades com pacientes de maior risco de desenvolvimento de LP, sendo, 14 enfermeiros da Clínica Médica, 14 da Clínica Cirúrgica, 20 da Unidade de Terapia Intensiva Adulto (UTI Adulto), 24 do Pronto Socorro Adulto (PSA), 10 da Pediatria e 15 da Unidade de Terapia Intensiva Pediátrica e Neonatal (UTI Pediátrica e Neonatal), incluindo os três turnos de trabalho. O PSA foi incluído, nesse estudo, em razão do tempo de permanência dos pacientes, quando não há leitos disponíveis para internação.

Cabe destacar que alguns enfermeiros deixaram de cumprir integralmente todas as fases do treinamento on-line sobre Prevenção de Lesão por Pressão, sendo descontinuados da pesquisa.

O treinamento on-line foi hospedado no ambiente virtual de aprendizagem Moodle ${ }^{\circledR}$, Modular Object-Oriented Dynamic Learning Environment, no website Cursos de Extensão da USP (http:/ / cursosextensao.usp.br).

Esse treinamento, com 12 horas e duração de quatro semanas, é composto por quatro módulos contendo fórum de dúvidas, aulas interativas, biblioteca com artigos científicos e guidelines, midiateca com vídeos, e exercícios de feedback, a saber: Módulo 01 - Por que prevenir? - abordado o custo do problema; Módulo 02 - Conceitos básicos - resgatou anatomia e fisiologia da pele; definição e fisiopatologia de LP, estágios das LP, Regiões vulneráveis para formação de LP, testes para identificação dos estágios das LP; Módulo 03 - Avaliação de risco - abordou os fatores de riscos extrínsecos e intrínsecos, avaliação do paciente, avaliação de risco, estudos de caso para aplicação da Escala de Braden e estudos de caso para avaliação dos fatores de risco; e, Módulo 04 - Protocolo de prevenção - contemplou vídeo STOP, lesão por pressão, elaboração de plano estratégico situacional.

Para representar a fisiopatologia, o estadiamento e as regiões vulneráveis à formação de LP, foi utilizado o Homem Virtual, ou seja, recurso computacional gráfico em terceira dimensão que potencializa a apreensão do conhecimento por meio de habilidades espaciais e visuais. ${ }^{30}$

As atividades realizadas foram: testes com fotos de LP para classificação dos estágios (exercício de múltipla escolha com feedback); três estudos de caso para a avaliação de fatores de risco para o desenvolvimento de LP e mais três para aplicação da Escala de Braden; elaboração de planejamento estratégico situacional de medidas preventivas; e fóruns de dúvidas em relação ao conteúdo do treinamento. Os estudos de caso foram elaborados a partir de situações reais. 
A qualidade do treinamento on-line, antes de ser aplicado, foi avaliada por 14 juízes das áreas de Estomaterapia, Design instrucional e Educação a Distância e Docência em Enfermagem, sendo realizado ajustes indicados pelos expertos.

O treinamento contou com quatro tutores, com objetivo de acompanhar, orientar e esclarecer possíveis dúvidas. Os tutores foram três enfermeiros estomaterapeutas, participantes do Grupo de Estomaterapia do HU/USP e uma enfermeira do Serviço de Ensino e Qualidade do HU/USP com mestrado na área de prevenção de LP.

O impacto em amplitude ou o efeito do treinamento no desempenho do enfermeiro foi medido por meiodo instrumento proposto e validado por Abbad, ${ }^{20}$ composto por 12 itens, em escala Likert, com as respostas variando na seguinte proporção: "discordo totalmente" (1) e "concordo totalmente" (5), ou seja, quanto maior a pontuação, maior o impacto em amplitude. O instrumento foi aplicado por meio de formulário eletrônico e respondido em até 45 dias do término do treinamento.

Para avaliar o Suporte à Transferência foi utilizado uma escala validada ${ }^{20}$ constituído por uma escala do tipo Likert, contendo 22 itens e graduação de 1 (sempre) a 5 (nunca) - sendo que a última questão foi excluída por não se aplicar ao treinamento de LP. A Escala foi enviada por e-mail, através de formulário eletrônico e respondida em até 45 dias do término do treinamento.

Este estudo seguiu as normas de ética em pesquisa envolvendo seres humanos, apresentadas na Resolução 196 de 1996 do Conselho Nacional de Saúde/Ministério da Saúde, sendo aprovado pelo Comitê de Ética em Pesquisa e pela Comissão de Pesquisa da USP (parecer de n. 059.318) sob o CAAE 29396714.3.0000.5392, e pelo Comitê de Ética em Pesquisa do HU/USP (parecer de n. 765.815) sob o CAAE 29396714.3.3001.0076.

A população do estudo foi convidada a participar do curso, sendo esclarecida sobre os objetivos e as finalidades da pesquisa, bem como sobre o sigilo, a privacidade e o anonimato de todos os participantes, assegurando os aspectos éticos do estudo. Os enfermeiros que participaram voluntariamente da pesquisa preencheram, através de meio eletrônico, pela plataforma Moodle ${ }^{\circledast}$, o Termo de Consentimento Livre e Esclarecido.

Os dados foram armazenados no software Excel $^{\circledR} \mathrm{e}$ as análises realizadas no software $\mathrm{R}^{\circledR}$ 3.2.2. ${ }^{31}$
As avaliações de Impacto em Amplitude e de Suporte à Transferência estão apresentadas em forma descritiva por item e de escore total, respectivamente, a fim de demonstrar cada item avaliativo como uma variável e representar uma variável global de impacto e de suporte.

Os escores de avaliação de Impacto em Amplitude e de Suporte à Transferência foram escalonados para variarem de 0 a 10 . Em outras palavras, os domínios estimados para cada indivíduo foram calculados pela seguinte equação:

$$
\text { Escore }=\frac{\bar{x}-\min (x)}{\max (x)-\min (x)} * 10
$$

Onde é a média das questões que compõem o domínio, $\min (x)$ é o mínimo valorpossível que pode ser encontrado como resposta para as questões, e analogamente, $\max (\mathrm{x})$ é o valor máximo. ${ }^{32}$

O coeficiente Alpha de Cronbach foi utilizado para verificar a consistência interna das escalas. ${ }^{33}$

\section{RESULTADOS}

A idade média dos participantes foi de 37,5 anos. Em relação ao gênero, as mulheres representaram $89,1 \%$ dos enfermeiros que participaram do treinamento. Quanto à formação dos enfermeiros, $42,2 \%$ possuíam graduação; $26,6 \%$ tinham realizado especialização lato sensu e 31,2\% haviam concluído o mestrado. As áreas de atuação dos enfermeiros variaram entre: oito $(12,5 \%)$ em UTI Pediátrica e Neonatal; 16 (25,0\%) em Pronto Socorro; nove (14,1\%) em Clínica Médica; 10 (15,6\%) em Clínica Cirúrgica; 12 (18,7\%) em UTI Adulto e em nove $(14,1 \%)$ em Pediatria.

Em relação à função, o grupo foi composto por 61 enfermeiros assistenciais $(95,3 \%)$ e três enfermeiros chefe $(4,7 \%)$.

A percepção de impacto do treinamento no trabalho foi avaliada positivamente, com média de $7,05 \pm 1,60$, e variação de 3,75 a 10,00.

A tabela 1mostra a frequência dos itens de avaliação de percepção de impacto por grau de concordância. $\mathrm{O}$ instrumento utilizado apresentou índice de consistência interna satisfatório (Alpha de Cronbach=0,901). 
Tabela 1 - Distribuição das respostas sobre Percepção de Impacto em Amplitude no Treinamento online sobre Prevenção de Lesão por Pressão. São Paulo, SP, Brasil, 2016. (n=35)

\begin{tabular}{|c|c|c|c|c|c|}
\hline \multirow[t]{2}{*}{ Item } & 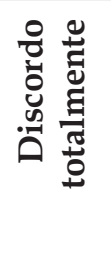 & 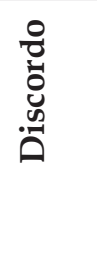 & 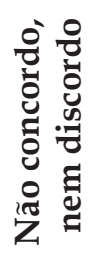 & 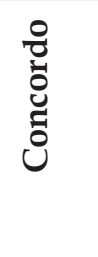 & 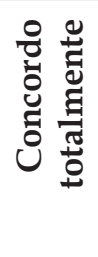 \\
\hline & $\begin{array}{r}n \\
(\%)\end{array}$ & $\begin{array}{r}n \\
(\%)\end{array}$ & $\begin{array}{r}n \\
(\%)\end{array}$ & $\begin{array}{r}n \\
(\%)\end{array}$ & $\begin{array}{r}n \\
(\%)\end{array}$ \\
\hline \multirow{2}{*}{$\begin{array}{l}\text { Utilizo com frequência em meu trabalho atual o que foi } \\
\text { ensinado no treinamento }\end{array}$} & 1 & 1 & 10 & 14 & 9 \\
\hline & 2,8 & 2,9 & 28,6 & 40 & 25,7 \\
\hline \multirow{2}{*}{$\begin{array}{l}\text { Aproveito as oportunidades que tenho para colocar em prática } \\
\text { o que me foi ensinado no treinamento }\end{array}$} & - & 2 & 3 & 16 & 14 \\
\hline & - & 5,7 & 8,6 & 45,7 & 40 \\
\hline \multirow{2}{*}{$\begin{array}{l}\text { As habilidades que aprendi no treinamento fizeram com que } \\
\text { eu cometesse menos erros em meu trabalho e em atividades } \\
\text { relacionadas ao conteúdo do treinamento }\end{array}$} & - & 2 & 7 & 15 & 11 \\
\hline & - & 5,7 & 20 & 42,9 & 31,4 \\
\hline \multirow[t]{2}{*}{ Recordo-me bem dos conteúdos ensinados no treinamento } & - & 2 & 12 & 14 & 7 \\
\hline & - & 5,7 & 34,3 & 40 & 20 \\
\hline \multirow{2}{*}{$\begin{array}{l}\text { Quando aplico o que aprendi no treinamento executo meu } \\
\text { trabalho com maior assertividade }\end{array}$} & - & 1 & 3 & 17 & 14 \\
\hline & - & 2,9 & 8,6 & 48,6 & 39,9 \\
\hline \multirow{2}{*}{$\begin{array}{l}\text { A qualidade do meu trabalho melhorou nas atividades } \\
\text { diretamente relacionadas ao conteúdo do treinamento }\end{array}$} & - & 1 & 5 & 20 & 9 \\
\hline & - & 2,9 & 14,3 & 57,1 & 25,7 \\
\hline \multirow{2}{*}{$\begin{array}{l}\text { A qualidade do meu trabalho melhorou mesmo naquelas } \\
\text { atividades que não pareciam estar relacionadas ao conteúdo } \\
\text { do treinamento }\end{array}$} & 1 & 3 & 13 & 13 & 5 \\
\hline & 2,9 & 8,6 & 37,1 & 37,1 & 14,3 \\
\hline \multirow{2}{*}{$\begin{array}{l}\text { Minha participação no treinamento serviu para aumentar } \\
\text { minha motivação para o trabalho }\end{array}$} & 2 & 2 & 16 & 4 & 11 \\
\hline & 5,8 & 5,7 & 45,7 & 11,4 & 31,4 \\
\hline \multirow{2}{*}{$\begin{array}{l}\text { Minha participação nesse treinamento aumentou minha } \\
\text { autoconfiança - Agora tenho mais confiança na minha } \\
\text { capacidade de executar meu trabalho com sucesso }\end{array}$} & 1 & - & 8 & 15 & 11 \\
\hline & 2,8 & - & 22,9 & 42,9 & 31,4 \\
\hline \multirow{2}{*}{$\begin{array}{l}\text { Após minha participação no treinamento tenho sugerido com } \\
\text { maior frequência mudanças nas rotinas de trabalho }\end{array}$} & - & 2 & 19 & 9 & 5 \\
\hline & - & 5,7 & 54,3 & 25,7 & 14,3 \\
\hline \multirow{2}{*}{$\begin{array}{l}\text { Esse treinamento que fiz me tornou mais receptivo a mudanças } \\
\text { no trabalho }\end{array}$} & 2 & 3 & 11 & 11 & 8 \\
\hline & 5,7 & 8,6 & 31,4 & 31,4 & 22,9 \\
\hline \multirow{2}{*}{$\begin{array}{l}\text { O treinamento que fiz beneficiou meus colegas de trabalho que } \\
\text { aprenderam comigo algumas novas habilidades }\end{array}$} & 1 & 3 & 12 & 12 & 7 \\
\hline & 2,8 & 8,6 & 34,3 & 34,3 & 20 \\
\hline
\end{tabular}

Quanto à percepção de impacto do treinamento no trabalho, $88,5 \%$ dos enfermeiros consideraram aplicar o que aprenderam no treinamento e executar o trabalho com maior assertividade.
A respeito da qualidade do trabalho, $82,8 \%$ dos enfermeiros afirmaram ter havido melhora nas atividades diretamente relacionadas ao conteúdo do treinamento. 
Na avaliação do Suporte à Transferência, exibida na tabela 2 , o suporte material teve a média mais alta, de 6,2 $\pm 2,04$, e os fatores situacionais de apoio mostram a falta de tempo para aplicar no trabalho o que o participante aprendeu, com o percentual de $57,2 \%$ de respostas. A média geral da escala de Suporte à Transferência foi de 5,82 $\pm 1,45$. O resultado do índice de consistência interna do instrumento utilizado foi satisfatório (Alpha de Cronbach=0,735).

Tabela 2 - Avaliação do Suporte à Transferência. São Paulo, SP, Brasil, 2016. (n=35)

\begin{tabular}{lcccccccc}
\hline \multicolumn{1}{c}{ Variáveis } & N & Mínimo & Máximo & Média & $\begin{array}{c}\text { Desvio } \\
\text { padrão }\end{array}$ & Mediana & $\begin{array}{c}\mathbf{1}^{\mathbf{o}} \\
\text { quartil }\end{array}$ & $\begin{array}{c}3^{\mathbf{0}} \\
\text { quartil }\end{array}$ \\
\hline $\begin{array}{l}\text { Fatores situacionais de } \\
\text { apoio }\end{array}$ & 35 & 1,67 & 8,06 & 5,68 & 1,55 & 6,11 & 4,17 & 6,94 \\
$\begin{array}{l}\text { Consequências } \\
\begin{array}{l}\text { associadas ao uso das } \\
\text { novas habilidades }\end{array}\end{array}$ & 35 & 1,43 & 8,93 & 5,72 & 1,50 & 5,71 & 4,82 & 6,61 \\
$\begin{array}{l}\text { Suporte material } \\
\text { Média geral }\end{array}$ & 35 & 2,00 & 10,00 & 6,20 & 2,04 & 6,50 & 5,00 & 7,25 \\
\hline
\end{tabular}

Dos achados referentes aos fatores situacionais de apoio, destacam-se: a falta de tempo para aplicar no trabalho o que o participante aprendeu, com $57,2 \%$ de respostas nas categorias "ocorre sempre" e "frequentemente"; e a alta demanda dos prazos para entrega de trabalhos que inviabilizam a aplicação das habilidades aprendidas no treinamento que apresentou $51,4 \%$ das respostas nos graus "ocorre sempre" e "frequentemente" (Tabela 3).

Tabela 3 - Respostas aos itens do questionário para a mensuração do Suporte à Transferência. São Paulo, SP, Brasil, 2016. (n=35)

Item

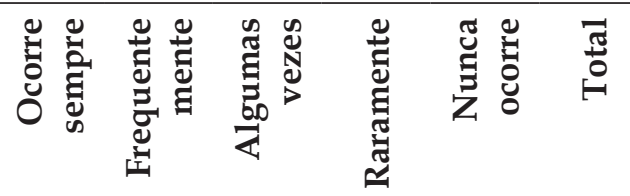

\begin{tabular}{rrrrrr}
$\mathbf{n}$ & $\mathrm{n}$ & $\mathrm{n}$ & $\mathrm{n}$ & $\mathrm{n}$ & $\mathrm{n}$ \\
$(\%)$ & $(\%)$ & $(\%)$ & $(\%)$ & $(\%)$ & $(\%)$ \\
\hline
\end{tabular}

Fatores situacionais de apoio

Tenho tido oportunidades de usar no meu trabalho as habilidades que aprendi no treinamento.

Falta-me tempo para aplicar no trabalho o que aprendi no treinamento.

Os objetivos de trabalho estabelecidos pelo meu chefe me encorajam a aplicar o que aprendi no treinamento.

Os prazos de entrega de trabalhos inviabilizam o uso das habilidades que eu aprendi no treinamento.

Tenho tido oportunidades de praticar habilidades importantes recém-adquiridas no treinamento - mas comumente pouco usadas no trabalho.

Os obstáculos e dificuldades associados a aplicação das novas habilidades que adquiri no treinamento são identificados e removidos pelo meu chefe.

$\begin{array}{rrrrrr}- & 2 & 4 & 16 & 13 & 35 \\ - & 5,7 & 11,4 & 45,7 & 37,2 & 100 \\ 3 & 17 & 11 & 4 & - & 35 \\ 8,6 & 48,6 & 31,4 & 11,4 & - & 100 \\ 1 & 5 & 8 & 13 & 8 & 35 \\ 2,8 & 14,3 & 22,9 & 37,1 & 22,9 & 100 \\ 2 & 16 & 15 & 2 & - & 35 \\ 5,7 & 45,7 & 42,9 & 5,7 & - & 100 \\ 3 & 7 & 9 & 12 & 4 & 35 \\ 8,6 & 20 & 25,7 & 34,3 & 11,4 & 100 \\ 2 & 10 & 9 & 9 & 5 & 35 \\ 5,7 & 28,6 & 25,7 & 25,7 & 14,3 & 100\end{array}$


Item

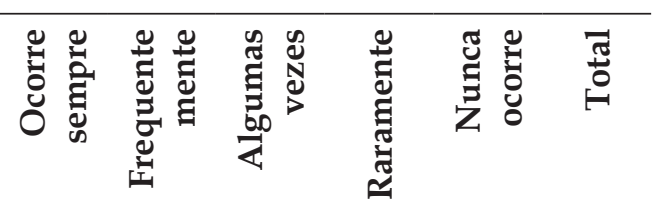

\begin{abstract}
Tenho sido encorajado pela minha chefia imediata a aplicar no meu trabalho o que aprendi no treinamento.

Consequências associadas ao uso das novas habilidades

Em meu ambiente de trabalho minhas sugestões em relação ao que foi ensinado no treinamento são levadas em consideração.

Eu recebo as informações necessárias à correta aplicação das novas habilidades no meu trabalho.
\end{abstract}

Meus colegas mais experientes apoiam as tentativas que faço de usar no trabalho o que aprendi no treinamento.

Aqui passam despercebidas minhas tentativas de aplicar no trabalho as novas habilidades que aprendi no treinamento.

Minha organização ressalta mais os aspectos negativos, por exemplo: lentidão dúvidas do que os positivos em relação ao uso das novas habilidades.

Tenho recebido elogios quando aplico corretamente no trabalho as novas habilidades que aprendi.

Quando tenho dificuldades em aplicar eficazmente as novas habilidades recebo orientações sobre como fazê-lo.

Chamam minha atenção quando cometo erros ao utilizar as habilidades que adquiri no treinamento.

Suporte material

Minha organização tem fornecido os recursos materiais, equipamentos materiais, mobiliário e similares necessários ao bom uso no trabalho das habilidades que aprendi no treinamento.

Os móveis materiais equipamentos e similares tem estado disponíveis em quantidade suficiente a aplicação do que aprendi no treinamento.

Os equipamentos e ou materiais por mim utilizados estão em boas condições de uso.

As ferramentas de trabalho, computadores, mesas de exame, sala de consulta, estetoscópio, otoscópio, por exemplo, são de qualidade compatível com o uso das novas habilidades.

O local onde trabalho no que se refere ao espaço mobiliário iluminação ventilação e ou nível de ruído e adequado a aplicação correta das habilidades que adquiri no treinamento.

\begin{tabular}{|c|c|c|c|c|c|}
\hline $\begin{array}{r}\mathbf{n} \\
(\%)\end{array}$ & $\begin{array}{r}n \\
(\%)\end{array}$ & $\begin{array}{r}n \\
(\%)\end{array}$ & $\begin{array}{r}n \\
(\%)\end{array}$ & $\begin{array}{r}n \\
(\%)\end{array}$ & $\begin{array}{r}n \\
(\%)\end{array}$ \\
\hline 2 & 6 & 6 & 9 & 12 & 35 \\
\hline 5,8 & 17,1 & 17,1 & 25,7 & 34,3 & 100 \\
\hline 1 & 2 & 9 & 16 & 7 & 35 \\
\hline 2,9 & 5,7 & 25,7 & 45,7 & 20 & 100 \\
\hline- & 4 & 9 & 14 & 8 & 35 \\
\hline- & 11,4 & 25,7 & 40 & 22,9 & 100 \\
\hline 1 & 2 & 9 & 16 & 7 & 35 \\
\hline 2,9 & 5,7 & 25,7 & 45,7 & 20 & 100 \\
\hline 1 & 1 & 16 & 13 & 4 & 35 \\
\hline 2,9 & 2,9 & 45,7 & 37,1 & 11,4 & 100 \\
\hline- & - & 14 & 10 & 11 & 35 \\
\hline- & - & 40 & 28,6 & 31,4 & 100 \\
\hline 4 & 11 & 12 & 7 & 1 & 35 \\
\hline 11,4 & 31,4 & 34,3 & 20 & 2,9 & 100 \\
\hline 3 & 5 & 14 & 9 & 4 & 35 \\
\hline 8,6 & 14,3 & 40 & 25,7 & 11,4 & 100 \\
\hline 10 & 11 & 11 & 2 & 1 & 35 \\
\hline 28,6 & 31,4 & 31,4 & 5,7 & 2,9 & 100 \\
\hline- & 4 & 10 & 15 & 6 & 35 \\
\hline- & 11,4 & 28,6 & 42,9 & 17,1 & 100 \\
\hline 1 & 3 & 11 & 13 & 7 & 35 \\
\hline 2,9 & 8,6 & 31,4 & 37,1 & 20 & 100 \\
\hline- & 5 & 6 & 17 & 7 & 35 \\
\hline- & 14,3 & 17,1 & 48,6 & 20 & 100 \\
\hline 2 & 5 & 11 & 13 & 4 & 35 \\
\hline 5,8 & 14,3 & 31,4 & 37,1 & 11,4 & 100 \\
\hline 4 & 4 & 17 & 7 & 3 & 35 \\
\hline 11,4 & 1,4 & 48,6 & 20 & 8,6 & 100 \\
\hline
\end{tabular}

As respostas predominantes dos participantes relacionadas ao item Consequências associadas ao uso das novas habilidades foram que as sugestões dos treinandos sobre o que foi ensinado no treinamento "nunca" e "raramente" foram levadas em consideração no ambiente de trabalho, para $65,7 \%$ 
dos enfermeiros, e em relação ao apoio de colegas mais experientes, $65,7 \%$ dos treinandos afirmaram que "nunca" e "raramente" são apoiados nas tentativas de usar no trabalho o que aprenderam no treinamento. Outro dado relevante é a falta de orientação para aplicar eficazmente as novas habilidades, sendo que $37,1 \%$ dos participantes alegaram que "nunca" e "raramente" recebem orientações sobre como fazer e $40 \%$ disseram receber "algumas vezes".

Em relação ao suporte material, 48,6\% dos enfermeiros afirmam que "algumas vezes" o local onde trabalho no que se refere ao espaço mobiliário, iluminação, ventilação e ou nível de ruído são adequadas a aplicação correta das habilidades que foram adquiridas no treinamento.

O Suporte à Transferência correlacionou-se significativamente $(0,374)$ com o Impacto em Amplitude e em profundidade no âmbito da autoavaliação pós-treinamento on-line.

\section{DISCUSSÃO}

Novas pesquisas sobre avaliações de treinamentos devem procurar medir o impacto na "assistência ao paciente", por representar um momento a partir do qual os benefícios significativos de quaisquer intervenções educativas possam ser julgados, para que se avalie, realmente, se o treinamento melhorou a qualidade e eficácia dos cuidados prestados. ${ }^{34 p r e-m o d u l e ~}$ data were collected on $74 \%$ ( $\mathrm{n}=29$ Assim, este estudo visou avançar na metodologia de avaliação de treinamento on-line, na área da saúde, por meio de um modelo proposto e utilizado pela psicologia organizacional, que avalia o impacto em amplitude, bem como o suporte à transferência do treinamento on-line. ${ }^{27}$

Desta forma, aponta-se a necessidade de investigar o ambiente pós-treinamento e buscar analisar as interferências que podem surgir e impactar nos resultados esperados do treinamento. ${ }^{11}$

O sistema de treinamento é composto pelos seguintes elementos: avaliação de necessidades de treinamento, planejamento e execução o de treinamento e avaliação de treinamento. A avaliação de treinamento é essencial para fornecer retroalimentações que assegurem o aperfeiçoamento constante do sistema de treinamento. ${ }^{35} \mathrm{O}$ processo avaliativo oportuniza reflexões que podem gerar mudanças, superações e evoluções. ${ }^{36}$

O presente estudo mostrou que houve transferência da aprendizagem para a prática assistencial de enfermagem, o que pode ser devido à pertinência da estratégia pedagógica utilizada, pois a transferência de conhecimentos para outra situação torna-se possível quando as estratégias de ensino-aprendizagem são aplicadas adequadamente. ${ }^{37} \mathrm{~A}$ avaliação do impacto de um treinamento presencial evidenciou que houve uma baixa retenção dos conteúdos aprendidos devido à aplicação de uma metodologia expositiva em excesso e em distanciamento com as situações de trabalho. ${ }^{20}$

O impacto em amplitude é medido em termos de transferência de aprendizagem e em relação à influência que a ação instrucional exerce sobre o desempenho global do treinando, em atividades específicas relacionadas ao objetivo da ação instrucional e da aplicação das habilidades aprendidas em situações não específicas ao treinamento. ${ }^{38}$ Os enfermeiros perceberam o impacto do treinamento on-line de forma positiva, evidenciando a aplicação de novos conhecimentos e habilidades em seu ambiente de trabalho e em tarefas não específicas ao treinamento.

O impacto em amplitude pode ser influenciado pela proximidade do conteúdo ensinado no treinamento em relação às situações reais de trabalho, ${ }^{39}$ nesse caso, a prevenção de LP é um cuidado frequente na assistência de enfermagem, ratificando a necessidade de aprendizagem e a aplicação prática mais assertiva por parte dos enfermeiros.

Dos achados referentes ao suporte à transferência, nos fatores situacionais de apoio, destacamse: a falta de tempo para aplicar no trabalho o que o participante aprendeu, e a alta demanda dos prazos para entrega de trabalhos que inviabilizam a aplicação das habilidades aprendidas no treinamento. Estudos apontam que a participação dos gerentes de enfermagem é fundamental no processo de gestão de pessoas evitando a sobrecarga de trabalho. ${ }^{40-41}$

Destaca-se ainda, a percepção do participante frente ao suporte permite investigar o apoio com o qual o treinando conta, ou julga contar, para aplicar o conhecimento na prática. ${ }^{18}$

Em relação à consequência predominante associada ao uso das novas habilidades, destaca-se a afirmativa de que as sugestões dos treinandos sobre o que foi ensinado no treinamento on-line, raramente, são levadas em consideração em seu ambiente de trabalho.

O suporte do supervisor e dos pares influencia significativamente na transferência do treinamento ${ }^{42} \mathrm{o}$ que não foi observado neste estudo, pois os enfermeiros responderam que não foram encorajados pela chefia imediata e não tiveram apoio dos colegas mais experientes.

O papel da liderança no acompanhamento e desenvolvimento do trabalhador da equipe de 
enfermagem deve ser considerado relevante, pois é o líder que fornece feedback dos pontos fortes e dos que necessitam de melhorias. ${ }^{20}$

Evidencia-se a importância do processo de supervisão, focado na dimensão educativa, que permite que haja mobilidade de conhecimento para áreas correlatas. Essa mobilização do conhecimento não está subordinada exclusivamente ao esforço individual, mas também ao investimento em qualificação, com experiências significativas para propiciar a reconversão profissional. ${ }^{43}$

Ressalta-se, aqui, o papel essencial que a liderança pode assumir, atuando como facilitador/ mentor e educador, no intuito de identificar se houve fixação da aprendizagem e a corresponsabilização como elemento fundamental na condução de mudança do comportamento. Dessa forma, é necessário incluir planos de ação para reforço da aprendizagem por meio de devolutivas, fazendo com que o treinando exponha suas reflexões a cerca do que apreendeu. ${ }^{20}$

Além disso, para que os profissionais possam aplicar seus conhecimentos, é imprescindível uma mudança organizacional, ${ }^{15}$ de modo que, o suporte material na assistência ao paciente com LP ou com risco para o desenvolvimento possa promover a prevenção e a melhoria da qualidade do tratamento da LP.

Esses achados corroboram as pesquisas que apontam o Suporte Psicossocial à Transferência como o grande responsável pelo Impacto do Treinamento no Trabalho (Impacto em Amplitude) e sugerem a realização de pesquisas que permitam identificar obstáculos à transferência de treinamento no trabalho e investigar diferentes estratégias póstreinamento que possam maximizar o uso das novas habilidades adquiridas. ${ }^{39}$

É imprescindível compreender a transferência e a construção de conhecimentos, bem como suas repercussões na dinâmica do trabalho e na produtividade dos colaboradores,quantitativa e qualitativamente, com o objetivo de verificar como a organização pode ser, além de um espaço de formação profissional, um espaço de saber, de produção e transmissão de conhecimentos. ${ }^{44}$

A maior dificuldade para avaliar o treinamento ocorre na dimensão dos resultados, devido à necessidade de se isolar as variáveis obtidas como resultados do treinamento, das demais variáveis organizacionais que possam ter influenciado esses resultados. $^{44}$

\section{CONCLUSÃO}

O treinamento on-line agregou conhecimento e a percepção de impacto do treinamento por parte dos enfermeiros indica sua contribuição para uma mudança mais ampla de conhecimento, habilidade e atitude dos enfermeiros adquiridos no treinamento on-line para a prática assistencial na prevenção de LP.

O suporte à transferência correlacionou-se significativamente com o impacto em amplitude pós-treinamento on-line.

A avaliação do suporte à transferência permitiu examinar o tipo de ambiente encontrado pelo enfermeiro para fazer com que os conteúdos aprendidos fossem transferidos para sua prática profissional. Permitiu, igualmente, compreender a identificação dos aspectos materiais e sociais, e ainda identificar a importância do respaldo da chefia para que novas habilidades adquiridas sejam colocadas em prática.

Esta pesquisa pode, nesse sentido, contribuir para que outras instituições realizem a avaliação de resultados de seus programas educativos, para além das avaliações de reação e aprendizagem, mais comumente empregadas, pode auxiliar, ainda, no desenvolvimento de outras pesquisas sobre avaliação de treinamentos na área de Enfermagem e da Saúde.

Para tanto, é necessário aperfeiçoar todo o processo de desenvolvimento de treinamento on-line, desde o primeiro momento que se dá no levantamento e avaliação da necessidade de temas a serem abordados para o treinamento, até a identificação da melhora na qualidade da assistência por longo prazo.

É fundamental a inserção de mecanismos apropriados para diagnóstico de necessidades e avaliação dos programas de treinamento e desenvolvimento do pessoal de enfermagem, visando impulsionar transformações nas instituições de saúde dentro de uma visão crítica e alicerçada na realidade, resultando na construção de conhecimento para a organização, para a profissão e para a sociedade.

\section{REFERÊNCIAS}

1. Salum NC, Prado ML. Continuing education in the development of competences in nurses. Texto Contexto Enferm [Internet]. 2014 [cited 2017 Jan 18]; 23(2):301-8. Available from: http://www.scielo.br/ $\mathrm{pdf} / \mathrm{tce} / \mathrm{v} 23 \mathrm{n} 2 / 0104-0707-t c e-23-02-00301 . p d f$

2. CameloSHH, Angerami ELS. Professional competence: the building of concepts and strategies developed by health services and implications for nursing. Texto 
Contexto Enferm [Internet]. 2013 [cited 2017 Jan 18]; 22(2):552-60. Available from: http:/ / www.scielo.br/ $\mathrm{pdf} / \mathrm{tce} / \mathrm{v} 22 \mathrm{n} 2 / \mathrm{v} 22 \mathrm{n} 2 \mathrm{a} 34 . \mathrm{pdf}$

3. Queiroz FM, Aroldi JBC, Oliveira GDS de, Peres HHC, Santos VLCG. Venous ulcer and compression therapy for nurses: development of online course. Acta Paul Enferm [Internet]. 2012 [cited 2017 Jan 20]; 25(3):43540. Available from: http://www.scielo.br/pdf/ape/ v25n3/en_v25n3a18.pdf

4. Chetley A, Davies J, Trude B, McConnell H, Ramirez R. Improving health connecting people: the role of ICTs in the health sector of developing countries. Washington DC World Bank Information for Development Program [infoDev] [Internet] 2006 May 31; [cited 2017 Jan 20]. Available from: http://documents. worldbank.org/curated/en/234041468163474585/pd f/375210Improving0health01PUBLIC1.pdf

5. Zerbini $T$, Abbad G. Impacto de treinamento no trabalho via internet. RAE eletrônica [Internet]. 2005 [cited 2017 Jan 20]; 4(2):1-21. Available from: http:/ / rae.fgv.br/sites/rae.fgv.br/files/artigos/10.1590_ S1676-5648200500020002.pdf

6. Ossiannilsson E, Landgren L. Quality in e-learning a conceptual framework based on experiences from three international benchmarking projects. J Comput Assist Learn. 2012;28:42-51.

7. SteinSJ, Shephard K, Harris I. Conceptions of e-learning and professional development for e-learning held by tertiary educators in New Zealand. Br J Educ Technol. 2011;42(1):145-65.

8. Wilson A. Effective professional development for e-learning: what do the managers think? Br J Educ Technol. 2012; 43(6):892-900.

9. Zerbini T, Abbad G. Qualificação Profissional a distancia: avaliação da transferencia de treinamento. Paidéia [Internet]. 2010 [cited 2017 Feb 01]; 20(47):31323. Available from: http://www.scielo.br/pdf/ paideia/v20n47/a04v20n47.pdf

10. Paschoal AS, Mantovani MDF, Méier MJ. Percepçao da educaçao permanente, continuada e em serviço para enfermeiros de um hospital de ensino. Rev Esc Enferm USP [Internet]. 2007 [cited 2017 Feb 05]; 41(3):478-84. Available from: http://www.scielo.br/ pdf/reeusp/v41n3/19.pdf

11. Borges-Andrade JE, Abbad GS, Mourão L. Treinamento, desenvolvimento e educação em organizações e trabalho: fundamentos para gestão de pessoas. Porto Alegre (RS): Artmed; 2006.

12. Nadler L. The handbook of human resource development. New York (US): Wiley; 1984.

13. Borges-Andrade JE. Desenvolvimento de medidas em avaliação de treinamento. Estud Psicol [Internet]. 2002 [cited 2017 Feb 05]; 7(Spe):31-43. Available from: http:/ / www.scielo.br/pdf/epsic/v7nspe/a05v7esp.pdf

14. Abbad GS, Borges-Andrade JE. Aprendizagem humana em organizações de trabalho. In: Zanelli JC, Borges-Andrade JE, Bastos AVB, editors. Psicologia, organizações e trabalho no Brasil. Porto Alegre (RS): Artmed; 2004. p. 237-75.

15. Otrenti E, Mira VL, Bucchi SM, Borges-andrade JE. Evaluation of formal educational processes for healthcare professionals. Invest Educ Enferm [Internet]. 2014 [cited 2017 Feb 08]; 32(2):103-11. Available from: http://www.scielo.org.co/pdf/iee/ v32n1/v32n1a12.pdf

16. Goldstein IL. Training in work organizations. In: Dunnette MD, Hough LM, editors. Handbook of industrial and organizational psychology. Palo Alto, CA (US): Consulting Psychologists Press; 1991. p. 507-619.

17. Abbad G, Gama ALG, Borges-Andrade JE. Treinamento: análise do relacionamento da avaliação nos níveis de reação, aprendizagem e impacto no trabalho. Rev Adm Contemp [Internet]. 2000 [cited 2017 Feb 08]; 4(3):25-45. Available from: http:/ / www. scielo.br/pdf/rac/v4n3/v4n3a03.pdf

18. Abbad G. Um modelo integrado de avaliação do impacto de treinamento no trabalho - IMPACT [tese]. Brasília (DF). Universidade de Brasília; 1999.

19. Alavarce DC. Desenvolvimento e avaliação da reação, aprendizagem e impacto de treinamento on-line para profissionais da saúde [tese]. São Paulo (SP). Universidade de São Paulo; 2014 [cited 2017 Feb 20]. Available from: http://www.teses.usp.br/teses/ disponiveis/7/7140/tde-17122014-111420/pt-br.php

20. Bastos LFL. Avaliação da reação, aprendizagem e impacto de treinamento em um hospital do município de São Paulo [dissertação]. São Paulo (SP). Universidade de São Paulo; 2012 [cited 2017 Feb 20]. Available from: http://www.teses.usp.br/teses/ disponiveis/7/7140/tde-03012013-175327/en.php

21. Mira VL. Avaliação de programas de treinamento e desenvolvimento da equipe de enfermagem de dois hospitais do município de São Paulo. [livre docência]. São Paulo (SP). Universidade de São Paulo; 2010 [cited 2017 Feb 22]. Available from: http:/ / www.teses.usp. $\mathrm{br} /$ teses/disponiveis/livredocencia/7/tde-14022012100136/en.php

22. Otrenti E. Avaliação de processos educativos formais para profisisonais da área da saúde: revisão integrativa de literatura [dissertação]. São Paulo (SP). Universidade de São Paulo; 2011[cited 2017 Feb 22]. Available from: http://www.teses.usp.br/teses/ disponiveis/7/7140/tde-20062011-151612/pt-br.php

23. Santos PT. Avaliação da satisafação, aprendizagem e impacto do curso introdutório à estratégia Saúde da família: um aporte metodológico [dissertação]. São Paulo (SP). Universidade de São Paulo; 2012 [cited 2017 Feb 22]. Available from: http://www.teses.usp.br/teses/ disponiveis/7/7140/tde-16022012-185757/pt-br.php

24. Cox MJ, Marshall G. Effects of ICT: Do we know what we should know? Educ Inf Technol. 2007; 12(2):59-70.

25. Bloomfield JG, While AE, Roberts JD. Using computer assisted learning for clinical skills education in nursing: integrative review. J Adv Nurs [Internet]. 
2008 [cited 2017 Feb 26]; 63(3):222-35. Available from: http:/ / www.ncbi.nlm.nih.gov/pubmed/18702768

26. Alvarez AG, Dal Sasso GTM. Virtual learning objects: contributions to the learning process in health and nursing. Acta Paul Enferm [Internet]. 2011 [cited 2017 Feb 22]; 24(5):707-11. Available from: http://www. scielo.br/pdf/ape/v24n5/en_18v24n5.pdf

27. Abbad G, Mourão L, Meneses PPM, Zerbini T, BorgesAndrade JE, Vilas-Boas R. Medidas de Avaliação em treinamento, desenvolvimento e educação: ferramentas para gestão de pessoas. Porto Wlegre (RS): Artmed; 2012.

28. Niederhauser A, VanDeusen Lukas C, Parker V, Ayello E a, Zulkowski K, Berlowitz D. Comprehensive programs for preventing pressure ulcers: a review of the literature. Adv Skin Wound Care [Internet]. 2012 [cited 2017 Feb 26]; 25(4):167-88. Available from: http:/ / www.ncbi.nlm.nih.gov/pubmed/22441049

29. Rogenski NMB, Kurcgant P. The incidence of pressure ulcers after the implementation of a prevention protocol. Rev Latino-am Enferm [Internet]. 2012 [cited 2017 Feb 20]; 20(2):1-7. Available from: http:/ / www. scielo.br/pdf/rlae/v20n2/16.pdf

30. Costa JB, Peres HHC, Rogenski NMB, Baptista CMC. An educational proposal to teach a pressure ulcer management course online to students and nursing professionals. Acta Paul Enferm [Internet]. 2009 [cited 2017 Feb 14]; 22(5):607-11. Available from: http:/ / www.scielo.br/pdf/ape/v22n5/en_02.pdf

31. R Core Team. R: A language and environment for statistical computing [Internet]. Vienna (AT): R Foundation for Statistical Computing; 2015 [cited 2017 Feb 10]. Available from: http:/ / www.r-project.org

32. Bussab W, Morettin P. Estatística básica. 6 ed. São Paulo (SP): Saraiva; 2006.

33. Cronbach L. Coefficient alpha and the internal structure of tests. Psychometrika [Internet]. 1951 [cited 2017 Feb 08]; 16(3):297-334. Available from: https:/ / www.psychometricsociety.org/sites/default/files/ cronbach_citation_classic_alpha.pdf

34. Wilkinson A, Forbes A, Bloomfield J, Gee CF. An exploration of four web-based open and flexible learning modules in post-registration nurse education. Int J Nurs Stud. 2004; 41(4):411-24.

35. Borges-Andrade JE, Abbad G. Treinamento e desenvolvimento: reflexões sobre suas pesquisas científicas. Rev Adm. 1996; 31(2):112-25.
36. Albertino FMDF, Souza NA. Avaliação da Aprendizagem: o portfólio como auxiliar na construção de um profissional reflexivo. Est Aval Educ [Internet]. 2004 [cited 2017 Mar 01]; (29):169-90. Available from:http://publicacoes.fcc.org.br/ojs/ index.php/eae/article/view/2164/2121

37. Zabala A, Arnau L. Como aprender e ensinar competências. Porto Alegre (RS): Artmed; 2010. 17-26 p.

38. Zerbini T, Abbad G. Transferência de treinamento e impacto do treinamento no trabalho: análise crítica da literatura. Rev Psi: Org e Trab [Internet]. 2010 [cited 2017 Feb 12]; 10(2):97-111. Available from:http:/ / pepsic.bvsalud.org/pdf/rpot/v10n2/v10n2a08.pdf

39. Tamayo N, Abbad G. Autoconceito profissional e suporte à transferência e impacto do treinamento no trabalho. Rev Adm Contemp [Internet]. 2006 [cited 2017 Mar 02]; 10(3):9-28.Available from: http:/ / www. scielo.br/pdf/rac/v10n3/a02v10n3.pdf

40. Meneghini F, Paz AA, Lautert L. Fatores ocupacionais associados aos componentes da síndrome de burnout em trabalhadores de enfermagem. Texto Contexto Enferm [Internet]. 2011 [cited 2017 Mar 05]; 20(2):22533. Available from: http://www.scielo.br/pdf/tce/ v20n2/a02v20n2.pdf

41. Novaretti MCZ, Santos EDV, Quitério LM, Daudgallotti RM. Sobrecarga de trabalho da Enfermagem e incidentes e eventos adversos em pacientes internados em UTI. Rev Bras Enferm [Internet]. 2014 [cited 2017 Mar 06]; 67(5):692-9. Available from: http:/ / www.scielo.br/pdf/reben/v67n5/0034-7167reben-67-05-0692.pdf

42. Burke LA, Hutchins HM. Training transfer: an integrative literature review. Hum Resour Dev Rev [Internet]. 2007 [cited 2017 Feb 10]; 6(3):26396. Available from: http://hrd.sagepub.com/cgi/ doi/10.1177/1534484307303035

43. Arruda MCC, Marteleto RM, Souza DB. Educação, trabalho e o delineamento de novos perfis profissionais: o bibliotecário em questão. Ci Inf [Internet]. 2000 [cited 2017 Feb 12]; 29(3):14-24. Available from: http:/ / www.scielo.br/pdf/ci/v29n3/a02v29n3.pdf

44. Scorsolini-comin F, Inocente DF, Miura IK. Avaliação de programas de treinamento, desenvolvimento e educação no contexto organizacional: modelos e perspectivas. Rev Psi Organ e Trab [Internet]. 2011 [cited 2017 Feb 12]; 11(1):37-53. Available from: http://pepsic.bvsalud.org/pdf/rpot/v11n1/ v11n1a04.pdf

Recebido: 20 de abril de 2017

Aprovado: 08 de novembro de 2017 This is an Open Access article distributed under the terms of the Creative Commons (CC BY).
Departamento de Orientação Profissional

Av. Dr. Enéas de Carvalho Aguiar, 419

05403-000 - Cerqueira César, São Paulo, SP, Brasil

E-mail: juscilynne@gmail.com 